\title{
The role of short-chain dehydrogenase/ oxidoreductase, induced by salt stress, on host interaction of $B$. pseudomallei
}

\author{
Pornpan Pumirat ${ }^{1}$, Usa Boonyuen${ }^{2}$, Muthita Vanaporn ${ }^{1}$, Peechanika Pinweha ${ }^{3}$, Sarunporn Tandhavanant ${ }^{1,4}$,
} Sunee Korbsrisate ${ }^{3}$ and Narisara Chantratita ${ }^{1,4^{*}}$

\begin{abstract}
Background: Burkholderia pseudomallei is the causative agent of melioidosis, a frequently occurring disease in northeastern Thailand, where soil and water high in salt content are common. Using microarray analysis, we previously showed that $B$. pseudomallei up-regulated a short-chain dehydrogenase/oxidoreductase (SDO) under salt stress. However, the importance of SDO in B. pseudomallei infection is unknown. This study aimed to explore the function of B. pseudomallei SDO, and to investigate its role in interactions between B. pseudomallei and host cells.

Results: Bioinformatics analysis of $B$. pseudomallei SDO structure, based on homology modeling, revealed a NAD cofactor domain and a catalytic triad containing Ser149, Tyr162, and Lys166. This is similar to Bacillus megaterium glucose 1-dehydrogenase. To investigate the role of this protein, we constructed a B. pseudomallei SDO defective mutant, measured glucose dehydrogenase $(\mathrm{GDH})$ activity, and tested the interactions with host cells. The $B$. pseudomallei K96243 wild type exhibited potent GDH activity under condition containing $300 \mathrm{mM} \mathrm{NaCl}$, while the mutant showed activity levels 15 times lower. Both invasion into the A549 cell line and early intracellular survival within the J774A.1 macrophage cell were impaired in the mutant. Complementation of SDO was able to restore the mutant ability to produce GDH activity, invade epithelial cells, and survive in macrophages.

Conclusions: Our data suggest that induced SDO activity during salt stress may facilitate B. pseudomallei invasion and affect initiation of successful intracellular infection. Identifying the role of B. pseudomallei SDO provides a better understanding of the association between bacterial adaptation and pathogenesis in melioidosis.
\end{abstract}

\section{Background}

Burkholderia pseudomallei is a Gram-negative bacillus and the causative agent of melioidosis, a severe disease endemic in Southeast Asia and northern Australia [1]. The organism is an environmental saprophyte found in soil and water. It infects humans and animals mostly by direct contact with wet soil $[1,2]$. The incidence of melioidosis is high in northeastern Thailand, where saline soil and water are abundant $[3,4]$. The salt concentration in soil in this region ranges from 40 to $1,000 \mathrm{mM}$ $\mathrm{NaCl}$ - significantly higher than the $20 \mathrm{mM} \mathrm{NaCl}$ average

\footnotetext{
* Correspondence: narisara@tropmedres.ac

'Department of Microbiology and Immunology, Faculty of Tropical Medicine, Mahidol University, Bangkok 10400, Thailand

${ }^{4}$ Mahidol-Oxford Tropical Medicine Research Unit, Faculty of Tropical

Medicine, Mahidol University, Bangkok 10400, Thailand

Full list of author information is available at the end of the article
}

in other parts of the country (Development Department, Ministry of Interior, Thailand). It has been suggested that high salt or osmotic stress in northeast Thailand may be a key factor for B. pseudomallei alteration for survival in the natural environment, and it may enable the bacteria to establish the infection in respective hosts. The relationship between high salt concentration and susceptibility to bacterial infection is described in cystic fibrosis (CF) patients [5]. The lung airway surface liquid of $\mathrm{CF}$ sufferers has twice the $\mathrm{NaCl}$ concentration of healthy lungs [6]. Opportunistic infections of CF lungs have been linked with a variety of pathogens, including B. cepacia complex $[7,8]$ and B. pseudomallei [9]. However, the impact of salt and osmotic stress on B. pseudomallei and the related mechanisms underlying B. pseudomallei pathogenesis in CF patients are unknown.

\section{Biomed Central}

\author{
the article
} (c) 2014 Pumirat et al.; licensee BioMed Central Ltd. This is an Open Access article distributed under the terms of the Creative
Commons Attribution License (http://creativecommons.org/licenses/by/2.0), which permits unrestricted use, distribution, and waiver (http://creativecommons.org/publicdomain/zero/1.0/) applies to the data made available in this article, unless otherwise 
An earlier study demonstrated that the killing efficiency of Burkholderia species, including B. pseudomallei, against the nematode Caenorhabditis elegans is enhanced in condition containing $300 \mathrm{mM} \mathrm{NaCl}$ [10]. We also showed that B. pseudomallei grown under salt stress invades a lung epithelial cell line A549 [11] more efficiently, and exhibits significantly greater resistance to ceftazidime, an antibiotic used to treat melioidosis [12]. Our transcriptional analysis revealed B. pseudomallei pre-exposed to salt stress up-regulates a 10-fold increase of a gene associated with short-chain dehydrogenase/oxidoreductase (SDO) [11]. A different study by Bhatt \& Weingart [13] also showed that an oxidoreductase encoding gene $(b s r A)$ was up-regulated in $B$. cenocepacia in response to increased $\mathrm{NaCl}$ concentrations. However, the role of SDO for B. pseudomallei adaptation to osmotic or salt stress remains unknown.

In the present study, we analyzed the protein sequence and predicted structure of B. pseudomallei SDO using bioinformatics analysis, to provide information about the possible functions of SDO. We further investigated its functional roles by constructing a SDO deletion mutant strain, and examined the interaction between mutant and host cells. The results suggest that SDO is an adaptive determinant of $B$. pseudomallei virulence, which is up-regulated under salt stress, and has a significant role in the interaction with host cells.

\section{Results}

\section{Bioinformatics analysis of $B$. pseudomallei SDO}

A SDO amino-acid (aa) sequence of B. pseudomallei strain K96243 was retrieved from GenBank (NCBI Reference Sequence: YP_112245.1; locus_tag = "BPSS2242" [14]). It was composed of 271 aa with a calculated molecular weight of 28,766 Dalton. BLAST [15] sequence analysis [16] revealed that B. pseudomallei SDO was categorized into short-chain dehydrogenases/reductases (SDRs), which shared a $24 \%$ amino-acid sequence identity with Bacillus megaterium glucose 1-dehydrogenase (PDB ID: 1GCO) (Figure 1A). Therefore, the SWISSMODEL [17] was used to construct a structural model of $B$. pseudomallei SDO, using B. megaterium glucose 1-dehydrogenase as a template for homology modeling. The resulting model was validated by PROCHECK [18]. The structural model of B. pseudomallei SDO revealed a catalytic triad active site, consisting of Ser149, Tyr162, and Lys166, together with a $\mathrm{NAD}^{+}$cofactor domain (Figure 1B). This suggests that the SDO of B. pseudomallei may have an enzymatic function similar to $B$. megaterium glucose 1-dehydrogenase.

Among available genomes of Burkholderia spp., BLAST analysis demonstrated that all species harbor the SDO protein. The amino-acid identities of pathogenic B. pseudomallei, B. mallei, B. oklahomensis, B. multivorans, B. vietnamiensis, and B. cenocepacia range from $83 \%$ to
$100 \%$, whereas those of non-pathogenic B. thailandensis are less than $36 \%$. The high identity among pathogenic strains might indicate a common pathogenesis that is mediated by Burkholderia SDO.

\section{Mutagenesis of $B$. pseudomallei SDO mutant}

To identify the function of SDO in B. pseudomallei, we constructed a mutant defective in SDO production using a pEXKm5-based allele replacement system [19]. PCR analysis using primers flanking deleted alleles confirmed the deletion of the SDO gene on the B. pseudomallei chromosome (Additional file 1). As expected, a 566 bp DNA fragment was detected in the SDO mutant, whereas a 1,197 bp DNA fragment was detected in the wild type K96243, indicating a homologous recombination by deletion of $631 \mathrm{bp}$ of the SDO gene on the chromosome of the B. pseudomallei mutant. B. pseudomallei SDO complement strain was constructed using the same strategy. The amplified product of the complemented strain showed the presence of a 1,197 bp DNA fragment, as observed in the parental strain. PCR analysis of a $236 \mathrm{bp}$ oriT fragment demonstrated an extinction of pEXKm5 plasmid backbone in both the mutant and complement strains.

The pEXKm5 plasmid was removed from the SDO mutant and the complement strains by sucrose selection. Absence of a $236 \mathrm{bp}$ oriT amplicon indicated the removal of pEXKm5 plasmid from the chromosome of the B. pseudomallei SDO mutant and the complement strains.

\section{B. pseudomallei SDO exhibits GDH activity under salt stress}

B. pseudomallei is known to up-regulate SDO in high salt condition [11]. The structural model of B. pseudomallei SDO indicates a catalytic triad and cofactor binding domain, similar to the structure of $B$. megaterium glucose 1-dehydrogenase. This is highly specific to betaD-glucose and is capable of using either $\mathrm{NAD}^{+}$or $\mathrm{NADP}^{+}$as a cofactor [20]. We hypothesized that the glucose dehydrogenase activity of $B$. pseudomallei SDO might be similar to $B$. megaterium. We determined the GDH activity of $B$. pseudomallei SDO in wild type and SDO mutant strains grown in LB broth containing 0$300 \mathrm{mM} \mathrm{NaCl}$. The results showed that B. pseudomallei wild type exhibited strong GDH activity under high salinity at $300 \mathrm{mM} \mathrm{NaCl}$, whereas the activity of B. pseudomallei was comparable in salt-free and $150 \mathrm{mM} \mathrm{NaCl}$ (Table 1). This correlated with previous finding that suggested B. pseudomallei SDO transcription was enhanced by salt stress [11].

It was also evident that the GDH activity of SDO mutant was impaired under high salt concentration condition containing $300 \mathrm{mM} \mathrm{NaCl}$ (Table 1), which was 15-fold lower than the wild type ( $p$-value $\leq 0.0001)$. The SDO complement strain was able to recover SDO mutant GDH activity (Table 1). The data suggested that high salt concentration 


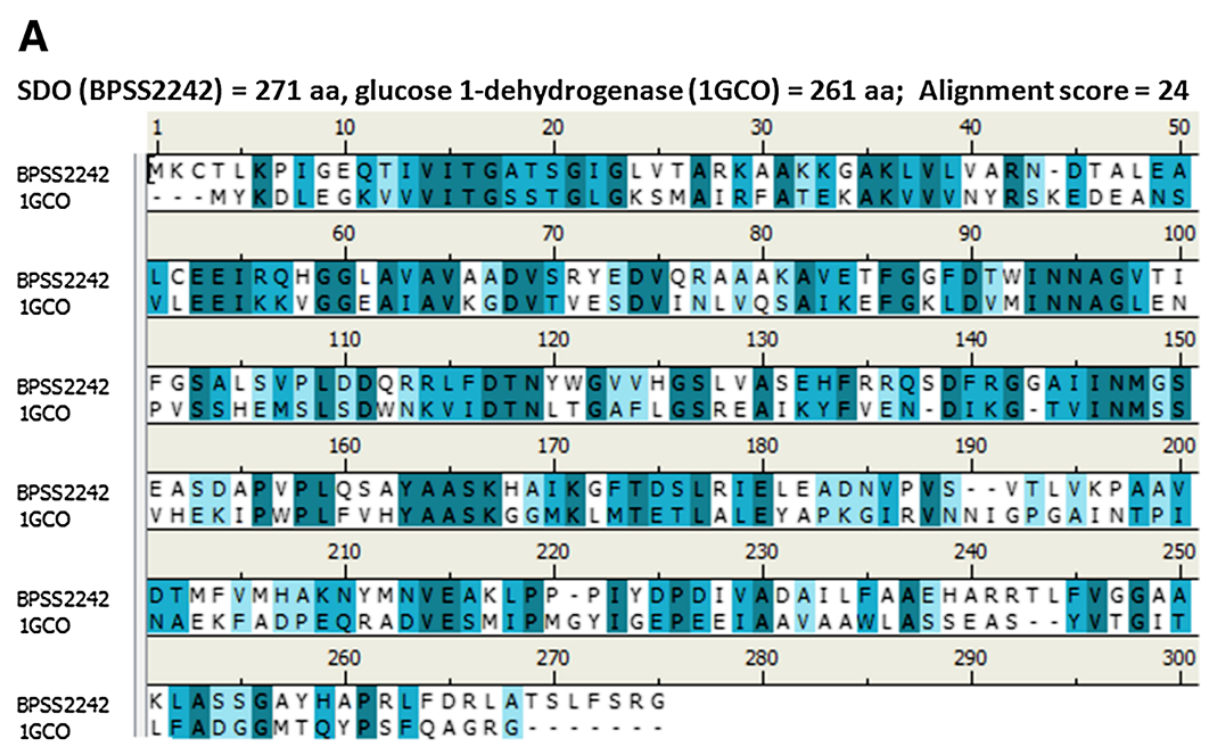

B

\section{B. pseudomallei \\ SDO}
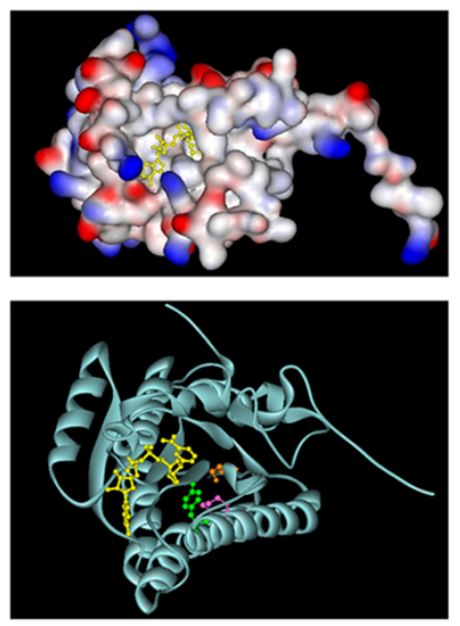

Catalytic Triad: Ser149, Tyr162, Lys 166
B. megaterium Glucose 1-dehydrogenase
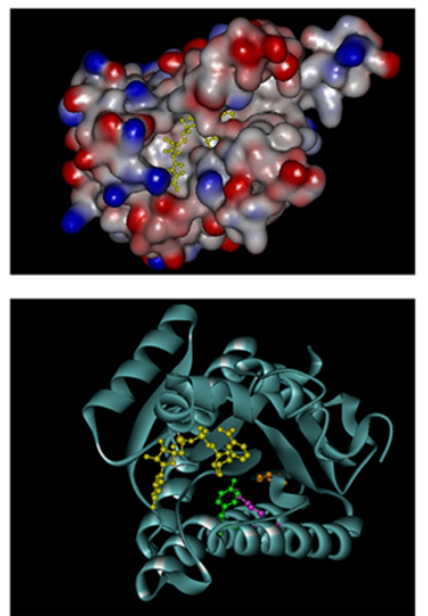

Catalytic Triad: Ser145, Tyr158, Lys 162

Figure 1 Protein sequence and structural comparison between B. pseudomallei SDO and B. megaterium glucose 1-dehydrogenase. A) Sequence alignment between B. pseudomallei SDO and B. megaterium glucose 1-dehydrogenase. B) Structural model of B. pseudomallei SDO (left) and structure of $B$. megaterium glucose 1-dehydrogenase (right), with bound NAD (yellow) shown in both surface (top) and cartoon representations (bottom). B. pseudomallei SDO and B. megaterium glucose 1-dehydrogenase shared structural similarities with conserved catalytic triad, consisting of Tyr (green), Thr (pink) and Lys (orange). Figures were generated by Discovery Studio Visualizer - Accelrys.

Table 1 Effect of $\mathrm{NaCl}$ treatment on GDH activity by B. pseudomallei K96243, SDO mutant, and complement strains

\begin{tabular}{cccc}
\hline \multirow{2}{*}{$(\mathbf{m M})$} & \multicolumn{3}{c}{ GDH activity $\mathbf{~ m U / m g}$} \\
\cline { 2 - 4 } & $\mathbf{K 9 6 2 4 3}$ & SDO mutant & SDO complement \\
\hline 0 & $0.049 \pm 0.006$ & $0.045 \pm 0.003$ & $0.042 \pm 0.005$ \\
\hline 150 & $0.066 \pm 0.012$ & $0.050 \pm 0.027$ & $0.056 \pm 0.017$ \\
\hline 300 & $0.996 \pm 0.109$ & $0.067 \pm 0.026$ & $0.952 \pm 0.060$
\end{tabular}

Data represent mean \pm standard error (SE) of three experiments made in triplicate. is associated with induction of SDO-dependent GDH activity in B. pseudomallei.

SDO plays a role in host interaction of $B$. pseudomallei The ability of $B$. pseudomallei to invade and survive in host cells is an important process that contributes to the pathogenesis of melioidosis. Invasion of $B$. pseudomallei has been reported as being induced by exogenous salt [11], and previous study indicated that high salt concentration increases the expression of SDO [11]. We thus investigated 
whether SDO affects the invasion of B. pseudomallei into A549 human lung respiratory epithelial cells. We found that invasion efficiency into A549 cells was significantly reduced in the B. pseudomallei SDO mutant, compared to the wild type ( $p$-value $\leq 0.05)$ (Figure 2$)$. The invasion efficiency of the $B$. pseudomallei mutant grown in LB broth containing 0,150 , and $300 \mathrm{mM} \mathrm{NaCl}$ was less than $5 \%$, in contrast to that of wild type bacteria $(5.90 \%, 10.57 \%$, and $17.68 \%$, in $\mathrm{LB}$ with 0,150 , and $300 \mathrm{mM} \mathrm{NaCl}$, respectively). While the mutant had less invasion efficiency, the result clearly demonstrated that increasing salt concentration from 0 to 150 or $300 \mathrm{mM} \mathrm{NaCl}$ led to significantly improved invasion of B. pseudomallei mutant into A549 cells as it is observed for the wild type strain (Figure 2).

The ability of $B$. pseudomallei to survive and replicate intracellularly may be attributable to the successful evasion of cellular killing strategies. We next examined the intracellular survival of the $B$. pseudomallei wild type and the SDO mutant within macrophages. The macrophage cells were chosen for this experiment because B. pseudomallei can be uptaken and multiply within these cells, and resist their bactericidal response [21,22]. The mutant showed fewer intracellular bacteria within the J774A.1 macrophage cell line during the initial stages of infection - up to $6 \mathrm{hrs}(p$-value $\leq 0.05)$ (Figure 3$)$. The intracellular doubling time of the B. pseudomallei SDO mutant pre-exposure to 0,150 , and $300 \mathrm{mM} \mathrm{NaCl}$ was $41.83 \pm 1.71,45.41 \pm 2.66$, and $50.41 \pm 1.33 \%$. In contrast, the doubling time of the wild type bacteria was $32.50 \pm$ $4.29,36.39 \pm 1.44$, and $47.23 \pm 2.31 \%$ in LB with 0,150 , and $300 \mathrm{mM} \mathrm{NaCl}$. The SDO complement strain recovered the growth of the SDO mutant with a rate similar to the wild type at an early time. Our data suggests that SDO plays an important role during the early phase of $B$. pseudomallei infection. It is possible the mutagenesis of SDO

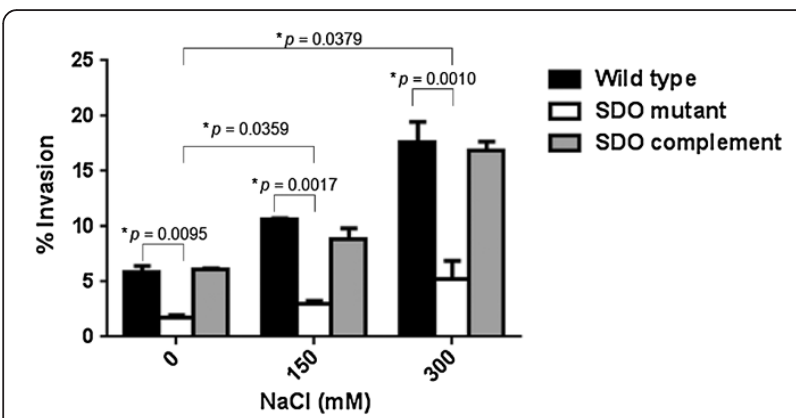

Figure 2 Invasion of A549 epithelial cells by B. pseudomallei. A549 cells were infected with overnight cultures of $B$. pseudomallei K96243 at MOI of 100, SDO mutant, and complement strains grown in $\mathrm{NaCl}$-free LB broth, LB broth with $150 \mathrm{mM} \mathrm{NaCl}$, or LB broth with $300 \mathrm{mM}$. Intracellular bacteria were counted after lysing infected cells at 4 hrs post-infection. Asterisks indicate significant differences ( $p$-value $\leq 0.05$, $t$-test) between groups. Error bars represent standard errors of mean for experiments performed in triplicate. impaired the invasion of B. pseudomallei into A549 epithelial cells, and delayed initial multiplication within J774A.1 macrophage cells.

\section{SDO is not essential for B. pseudomallei growth and colony morphotype}

Some dehydrogenases are critical for the growth characteristic of bacteria [23-25]. We investigated the effect of SDO deletion on the growth of B. pseudomallei. Growth of the wild type K96243 and the SDO mutant was compared in Luria-Bertani (LB) medium, containing various concentrations of $\mathrm{NaCl}(0,150,300$, and $450 \mathrm{mM})$. We observed that the growth kinetics of the B. pseudomallei K96243 and the SDO mutant were comparable (Figure 4A). The culture condition containing $450 \mathrm{mM} \mathrm{NaCl}$ impaired the growth of both strains. Variations in colony morphology are a notable feature of B. pseudomallei growth, where certain types are associated with enhanced bacterial survival under adverse conditions [26]. We also examined the effect of SDO on colony morphotype switching in the B. pseudomallei K96243 and the SDO mutant on Ashdown agar. The results indicated no phenotypic change of colony morphology between the wild type K96243 and the mutant. Both were categorized as colony morphotype I [26] (Figure 4B). These results indicated that SDO deletion does not affect $B$. pseudomallei colony morphology and bacterial growth.

\section{SDO is not required for $B$. pseudomallei survival under oxidative stress}

Many reports suggested that dehydrogenases are associated with the bacterial protection against toxic oxidants [27-33]. We examined the role of SDO for survival of $B$. pseudomallei under different oxidative stress conditions. Salt-treated and untreated B. pseudomallei wild type and SDO mutant strains were cultured on LB agar plates containing $250 \mu \mathrm{M} \mathrm{H}_{2} \mathrm{O}_{2}, 400 \mu \mathrm{M}$ menadione, or $200 \mu \mathrm{M}$ tert-butyl hydroperoxide $(\mathrm{tBOOH})$, and their survival were determined (Table 2). The result showed that there are no significant differences in survival between the B. pseudomallei wild type and the SDO mutant strains, neither in salt-treated or untreated conditions. This indicates that SDO might not be essential for adaptation and growth of $B$. pseudomallei in these oxidative stress environments.

\section{Discussion and conclusions}

B. pseudomallei is a saprophyte bacterium habitats in environments in endemic area, including Northeast Thailand [1] where saline salt and water are abundant. In normal physiological conditions, the $\mathrm{NaCl}$ concentration in the human lung is between 50 to $100 \mathrm{mM}$, and in the blood it can be as high as $150 \mathrm{mM}[34,35]$. In CF patients, the defective lung airway surface liquid has 

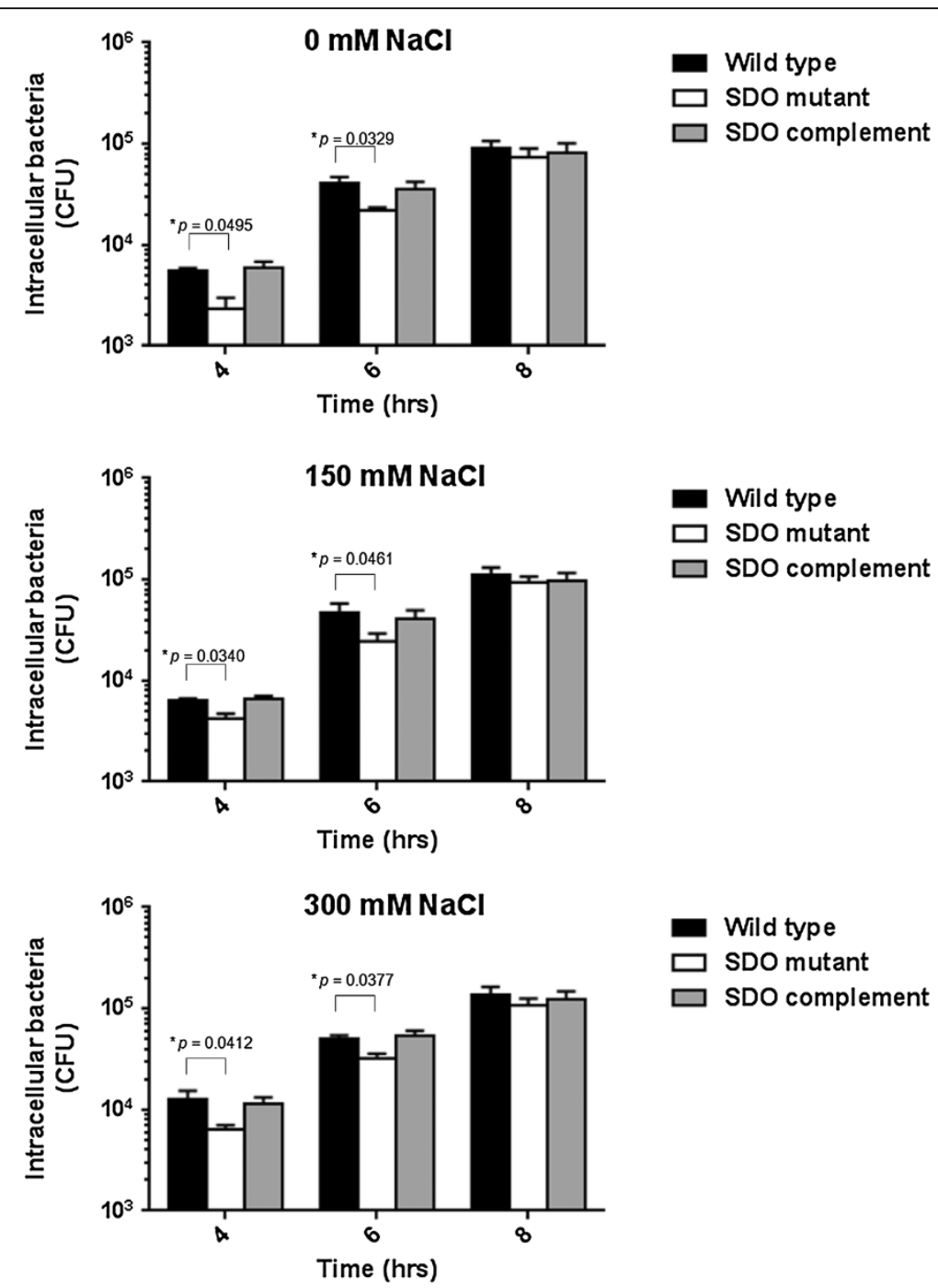

Figure 3 Intracellular survival of B. pseudomallei in J774A.1 macrophages. J774A.1 cells were infected with overnight cultures of B. pseudomallei K96243 at MOI of 2, SDO mutant and complement strain grown in NaCl-free LB broth, LB broth with $150 \mathrm{mM} \mathrm{NaCl}$, or LB broth with $300 \mathrm{mM}$. Intracellular bacteria were counted after lysing infected cells at 3, 6 and 9 hrs post-infection. Asterisks indicate significant differences ( $p$-value $\leq 0.05, t$-test) between groups. Error bars represent standard errors of mean for experiments performed in triplicate.

twice the $\mathrm{NaCl}$ concentration compared to healthy lungs $[6,34]$. It has been reported that elevated salt levels causes failure of bacterial killing in CF patients [5,6,34]. The opportunistic infection of CF lungs is linked to a variety of pathogens, including B. pseudomallei [7-9]. There is increasing evidence suggesting that salt concentration or osmolarity in a habitat influences the survival and pathogenicity of $B$. pseudomallei [10-12,36,37]. Thus, understanding the effect of salt stress is beneficial not only for environmental adaptation but also pathogenesis of the disease.

To survive in a high salt environment, the bacteria can undergo adaptation by altering the regulation of gene expression. Using transcriptomic analysis, we recently discovered that $B$. pseudomallei responds to salt stress by modulating the transcription of specific genes [11]. Among these are several loci associated with unknown functions, which need to be identified. Changes of B. pseudomallei transcriptome under salt stress include increasing expression of SDO [11]. The SDO is an enzyme in the short-chain dehydrogenases/reductases/oxidoreductase family that catalyzes the following chemical reaction: D-glucose $+\mathrm{NAD}^{+}=\mathrm{D}$-glucono-1,5-lactone $+\mathrm{NADH}+\mathrm{H}^{+}$. Both $\mathrm{NADP}^{+}$and $\mathrm{NAD}^{+}$are usually utilized as cofactors [38]. This study revealed the importance of SDO expression during salt-stress adaptation. Based on the structural model of B. pseudomallei SDO, which consists of a NAD ${ }^{+}$ cofactor domain and catalytic triad containing Ser149, Tyr162, and Lys166 similar to Bacillus megaterium glucose 1-dehydrogenase, we hypothesized that B. pseudomallei 


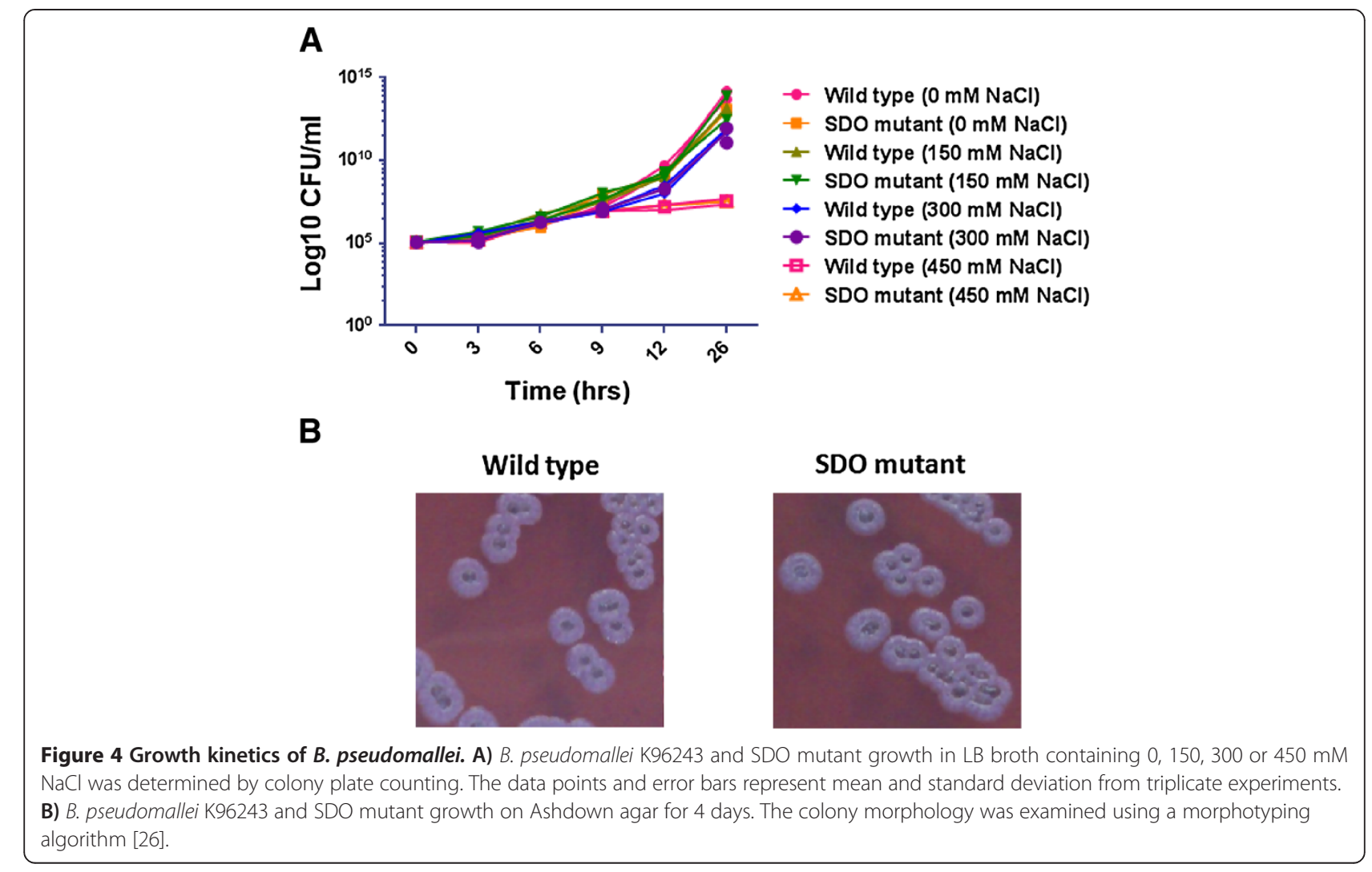

SDO has GDH activity. To examine the function of B. pseudomallei SDO, a mutant strain lacking SDO was constructed using a gene replacement strategy, a method that rarely has a polar effect on downstream genes [19]. In contrast to the wild type, it is clear that the B. pseudomallei SDO mutant was unable to produce GDH activity under high salt concentration. This finding is consistent with our previous observation of transcriptome profiling that B. pseudomallei grown in LB broth with $320 \mathrm{mM} \mathrm{NaCl}$ induced a 10-fold up-regulation of the SDO gene [11]. Since the mutant lost the gene encoding for functional SDO enzyme, it was thus unable to catalyze the reaction.
Several studies indicate that dehydrogenase enzymes are critical for bacterial growth. For instance, Brown \& Whiteley [23] have shown that the gene AA02749 (lctD), encoded for an $\mathrm{NAD}^{+}$-independent L-lactate dehydrogenase, is necessary for the growth of Aggregatibacter actinomycetemcomitans. Inactivation of the AA02769 gene affects the growth of the bacteria in the presence of L-lactate. Another study showed that mutations of the nuo gene encoding for NADH dehydrogenase I affected $E$. coli growth during the stationary phase culture in tryptone broth [24]. In our current study, we found that the B. pseudomallei mutant lacking SDO had growth kinetics and colony phenotypes similar to

Table 2 Effect of $\mathrm{NaCl}$ treatment on B. pseudomallei survival under oxidative stress conditions

\begin{tabular}{|c|c|c|c|c|c|}
\hline \multirow[t]{2}{*}{ B. pseudomallei } & \multirow[t]{2}{*}{$\mathrm{NaCl}(\mathrm{mM})$} & \multicolumn{4}{|c|}{$\%$ Bacterial survival } \\
\hline & & Control & $250 \mu \mathrm{M} \mathrm{H} \mathrm{O}_{2}$ & $400 \mu \mathrm{M}$ menadione & $200 \mu \mathrm{M}$ tBOOH \\
\hline \multirow[t]{3}{*}{ K96243 } & 0 & 100 & $58.6 \pm 4.3$ & $17.2 \pm 3.7$ & $62.6 \pm 2.4$ \\
\hline & 150 & 100 & $75.8 \pm 2.6$ & $31.0 \pm 3.4$ & $65.4 \pm 3.3$ \\
\hline & 300 & 100 & $82.8 \pm 3.9$ & $72.4 \pm 4.7$ & $68.9 \pm 5.5$ \\
\hline \multirow[t]{3}{*}{ SDO mutant } & 0 & 100 & $60.9 \pm 3.4$ & $17.8 \pm 2.9$ & $58.5 \pm 2.4$ \\
\hline & 150 & 100 & $72.7 \pm 4.0$ & $32.7 \pm 5.8$ & $64.0 \pm 3.9$ \\
\hline & 300 & 100 & $86.2 \pm 5.1$ & $75.8 \pm 6.2$ & $67.6 \pm 5.5$ \\
\hline
\end{tabular}

Data represent mean $\pm \mathrm{SE}$ of three experiments made in triplicate. 
the B. pseudomallei wild type. At various salt concentrations, there was no significant difference in growth between both $B$. pseudomallei strains. It indicated that deletion of the SDO gene has no effect on B. pseudomallei growth. This result is in agreement with previous observations identified by microarray analysis - the SDO gene is not in a group of growth-phase regulated genes [39].

The association between dehydrogenase enzymes and bacterial pathogenesis has been reported in several studies $[40,41]$. The alcohol acetaldehyde dehydrogenase (lmo1634), also known as Listeria adhesion protein, which is present in pathogenic Listeria species, mediates pathogenicity by promoting bacterial adhesion to enterocytelike Caco-2 cells [42]. It was shown that both lipoamide dehydrogenase "Lpd", a member of three multienzyme complexes in pyruvate dehydrogenase complex, and 3-ketosteroid 1(2)-dehydrogenase are important for virulence of Mycobacterium tuberculosis [43,44]. In Pseudomonas aeruginosa, the SDO attenuated mutant had significantly reduced pyocyanin production, motility, and biofilm formation, as well as absent paralysis of C. elegans [45]. Consistent with these reports, our study shows that defective SDO is associated with a reduced efficiency of the mutant to invade into A549 lung epithelial cells. Furthermore, we observed that the invasion of the B. pseudomallei SDO mutant was enhanced by increasing concentration of $\mathrm{NaCl}$ to 150 or $300 \mathrm{mM}$. Compared to the wild type, the SDO mutant exhibited fewer invasions and subsequently revealed less replication at early infection time point, but at $8 \mathrm{hrs}$ after infection the mutant was able to multiply in J774A.1 macrophage cells. The results suggest that the SDO gene might be induced only upon bacterial invasion of macrophage. It should be noted that B. pseudomallei grown under high salt conditions in vitro can up-regulate other virulence genes such as bsa T3SS. It is possible that this increased invasion was partly controlled by other salinity associated invasion- and virulence mechanisms, at least by coordinating regulation of the $b s a$ T3SS [11]. Previous studies have demonstrated that the mutant defect in bsa T3SS genes such as $b s a Z$ and bipD remained trapped in vesicles at earlier infection time points, but at 8 and 12 hrs after infection, the $b s a Q$ and $b s a Z$ mutants are able to escape into the cytosol and multiply effectively $[46,47]$. However, our finding in this study indicates that the SDO is involved in the pathogenesis of $B$. pseudomallei by facilitating the invasion and initial intracellular survival within host cells. It is feasible that SDO modulates the $\mathrm{NAD}^{+}$- or $\mathrm{NADP}^{+}$-dependent reaction associated with virulence expression when the $B$. pseudomallei encounters high salt stress.

The central role of bacterial defense against oxidative stress has been reported in many pathogenic bacteria $[30,48,49]$, especially during aerobic respiration and interactions with phagocytic cells. Several reports have indicated that bacterial dehydrogenases are important enzymes in oxidative stress response, such as NADH dehydrogenase, lactate dehydrogenase, formate dehydrogenase, succinate dehydrogenase, fumarate reductase, and glutathione-dependent formaldehyde dehydrogenase [27-32]. In Bacillus subtilis, two glucose dehydrogenases (YxnA and YcdF) assigned to a family of short-chain dehydrogenases are required for severe ethanol stress [33]. In our present study, we found no difference in bacterial counts between the SDO mutant compared to the wild type $B$. pseudomallei on LB agar plates containing various oxidative agents for both $\mathrm{NaCl}$-treated and untreated conditions. This indicates that SDO might not be crucial for B. pseudomallei to survive in oxidative stress environments. However, the survival under oxidative stresses increased in $\mathrm{NaCl}$ treated B. pseudomallei with higher concentrations, from $0 \mathrm{mM}$ to $150 \mathrm{mM}$, and up to $300 \mathrm{mM} \mathrm{NaCl}$ (Table 2). This finding suggests that $\mathrm{NaCl}$ may contribute to increase the oxidative stress tolerance of B. pseudomallei. Understanding the mechanism linking B. pseudomallei adaptation in saline environments to oxidative resistance requires further investigation.

In conclusion, our study revealed that B. pseudomallei SDO is involved in enhanced GDH activity in salt stress environments. The B. pseudomallei mutant lacking SDO had reduced abilities in invasion and initial intracellular survival. This indicates that this enzyme is associated with the pathogenesis of $B$. pseudomallei, especially when $B$. pseudomallei encounter salt stress. Due to the important role of SDO in pathogenesis, microbial SDOs might be a new target for the development of novel antibiotics. Thus, an understanding of the salt stress response of B. pseudomallei by the induction of SDO may provide important information in developing a new strategy for treatment of melioidosis.

\section{Methods}

Bacterial strains, growth conditions, and cell lines

B. pseudomallei wild type (K96243), the SDO mutant, and the complement strains were cultured in LuriaBertani (LB) medium and grown at $37^{\circ} \mathrm{C}$.

B. pseudomallei growth kinetics under stress conditions were performed as previously described [11]. The overnight culture of $B$. pseudomallei adjusted to $\mathrm{OD}_{600}$ 0.5 was inoculated 1:500 into $10 \mathrm{ml}$ of LB broth, with or without $\mathrm{NaCl}$ (Merck). Every 2 hrs after inoculation, the optical density of cultures at various time points was recorded, and serial dilution of these cultures was performed for colony-forming unit counts (CFU).

The cell lines A549 (human respiratory epithelial cell) and J774A.1 (mouse macrophage cell) were maintained in Dulbecco's Modified Eagle Medium (DMEM), 
which was supplemented with $10 \%$ heat-inactivated $\left(30 \mathrm{~min}, 56^{\circ} \mathrm{C}\right.$ ) fetal bovine serum (FBS), and incubated at $37^{\circ} \mathrm{C}$ in a humidified incubator in the presence of $5 \% \mathrm{CO}_{2}$.

\section{Bioinformatics analysis of $B$. pseudomallei SDO}

The B. pseudomallei SDO amino-acid sequence was subjected to basic local alignment search (BLAST) [15]; further alignment was then performed using ClustalW [16]. The sequence with maximum identity, Bacillus megaterium glucose 1-dehydrogenase, was used as a template for homology modeling using SWISS-MODEL [17]. The constructed model was validated by PROCHECK [18].

\section{Construction of B. pseudomallei SDO deletion mutant and complemented strain}

Deletion mutagenesis of the SDO gene was performed by homologous recombination (Additional file 1), as previously described by Lopez et al. [19]. The B. pseudomallei K96243 SDO gene sequence was obtained from GenBank (accession number NC_ 006351 and locus_tag = "BPSS2242" [14]). Primers used in this study were designed using Primer-BLAST (http://www.ncbi. nlm.nih.gov/tools/primer-blast). The primer sequences are shown in Table 3. Molecular cloning was carried out on 5' $298 \mathrm{bp}$ upstream and 3' $288 \mathrm{bp}$ downstream fragments of the B. pseudomallei SDO gene. The 5' upstream and 3' downstream fragments of the SDO gene were ligated by PCR using BPSS2242-F1 and BPSS2242-R2; this was facilitated by a tail on the 3' forward primer to give a new PCR product with a deletion in the region (631 bp) between BPSS2242-R1 and BPSS2242-F2.

This constructed fragment was cloned into $\mathrm{pGEM}^{\bullet}-\mathrm{T}$ Easy Vector and transformed into Escherichia coli strain DH $5 \alpha$. White colonies were selected using $\beta$-galactosidase indicator medium, using $50 \mu \mathrm{g} / \mathrm{ml}$ 5-bromo-4-chloro-3indolyl- $\beta$-D-galactopyranoside (X-Gal) (Promega) plates containing $100 \mu \mathrm{g} / \mathrm{ml}$ ampicillin. Colonies harboring the desired plasmid were analyzed by PCR using primers flanking the mutant allele (BPSS2242-F1 and BPSS2242-R2). Products were checked for correct size by agarose gel electrophoresis and verified by DNA sequencing.
The unmarked knockout cassette assembled by PCR containing the deletion of the SDO gene was cloned into the non-replicative plasmid, pEXKm5 [19]. The pEXKm5-mutant allele construct was then transformed into E. coli strain DH5 $\alpha$. Plasmids were extracted and checked by PCR, with primers BPSS2242-F1 and BPSS2242-R2, for correct product sizes of the target gene. The pEXKm5-mutant plasmid was transformed into $E$. coli strain RHO3 and delivered to the host B. pseudomallei strain $\mathrm{K} 96243$ by conjugation. This resulted in integration of the allelic replacement construct into the B. pseudomallei chromosome by homologous recombination between cloned and chromosomal sequences.

Conjugant clones grown on LB agar containing $1000 \mu \mathrm{g} / \mathrm{ml}$ kanamycin and $50 \mu \mathrm{g} / \mathrm{ml}$ 5-bromo-4-chloro3 -indolyl- $\beta$-D-glucuronide (X-Gluc) (Promega) were selected for PCR, with primers flanking the mutant allele (BPSS2242-F1 and BPSS2242-R2).

The conjugant clones were then streaked onto yeast extract tryptone (YT) agar (Yeast Extract \& Tryptone, BD; Agar, Oxoid) containing $15 \%$ sucrose and $50 \mu \mathrm{g} / \mathrm{ml}$ $\mathrm{X}$-Gluc, and incubated at $25^{\circ} \mathrm{C}$ for $72 \mathrm{hrs}$. The colonies growing on X-Gluc-containing medium (YT-sucrose-XGluc plate) were selected and purified by streaking on the same medium, and incubated as described above. Confirmation of deletion mutant was performed by PCR using primer sets flanking the mutant deletion allele primers (BPSS2242-F1 and BPSS2242-R2) and the oriT pEXKm5 plasmid backbone sequences.

Complement strains were constructed using the same pEXKm5-based allele replacement approach. Forward and reverse primers corresponding to the relevant regions of the genome sequences were amplified by BPSS2242-F1 and BPSS2242-R2 primers. The PCR amplicon (1,197 bp) contained the wild type $B$. pseudomallei SDO sequence. The construct was cloned into pEXKm5, transformed into E. coli $\mathrm{RHO}$, and delivered to the B. pseudomallei mutant by conjugation, resulting in merodiploid formation. Sucrose selection was employed for merodiploid resolution, resulting in the generation of wild type sequences, as well as strains that maintained the deletion alleles. PCR was performed with primers flanking deleted alleles to screen for strains that had the mutant allele replaced with the wild

Table 3 Oligonucleotide primers used for PCR

\begin{tabular}{cccc}
\hline Primer names & Oligo sequences (from 5'-3') & Purpose & Reference \\
\hline BPSS2242-F1 & ACCGCGCGACCGATATGAACG & Forward primer for upstream fragment of SDO gene & This study \\
\hline BPSS2242-F2 & GGACTCCTTGCCGAACGGGC & Reverse primer for upstream fragment of SDO gene & This study \\
\hline BPSS2242-R1 & GCCCGTTCGGAAGGAGTCC AACGTCGAGGCGAAGCTGCC & Forward primer for downstream fragment of SDO gene & This study \\
\hline BPSS2242-R2 & TCCCTTCGCGCTCGTGCAAC & Reverse primer for downstream fragment of SDO gene & This study \\
\hline OriT-F & CAGCCTCGCAGAGCAGGATTC & Forward primer for oriT & [50] \\
\hline OriT-R & TCCGCTGCATAACCCTGCTTC & Reverse primer for oriT & [50] \\
\hline
\end{tabular}


type sequence. PCR with oriT-specific primers [50] was used to demonstrate the absence of pEXKm5 plasmid backbone.

\section{GDH activity assay}

An overnight culture of B. pseudomallei wild type K96243, SDO mutant, and complement strains grown in salt-free LB broth, was subcultured 1:10 into LB broth containing 0,150 , or $300 \mathrm{mM} \mathrm{NaCl}$ and incubated at $37^{\circ} \mathrm{C}$ for $6 \mathrm{hrs}$. The bacteria cells were then examined by OD600 measurement and CFU plate counting, to confirm that they derived from cultures containing the same numbers of viable bacteria. B. pseudomallei wild type K96243, SDO mutant, and complement strains were all lysed with EasyLyse ${ }^{\mathrm{TM}}$ Bacterial Protein Extraction Solution (Epicentre, Madison, Wisconsin) to release intracellular proteins. The supernatant was separated from bacterial debris by centrifugation; protein concentration was then measured by BCA Protein Assay Kit (Pierce ${ }^{\oplus}$, Rockford, USA). GDH activity of $100 \mu \mathrm{g}$ of $B$. pseudomallei proteins, wild type K96243, SDO mutant, and complement, were determined in a microtiter plate using the GDH Activity Assay Kit (BioVision, Mountain View, USA) as described by the manufacturer. $100 \mu \mathrm{l}$ of reagent mixture containing assay buffer, developer, and glucose was briefly mixed with $50 \mu \mathrm{l}$ of protein samples, reagent control, or GDH positive controls. The OD values at $450 \mathrm{~nm}$ of the mixtures were measured before and after incubating for $1 \mathrm{hr}$ at $37^{\circ} \mathrm{C}$. The NADH standard curve was constructed to determine GDH activity (mU/mg).

Phenotypic examination of the B. pseudomallei SDO mutant Colony morphology of the B. pseudomallei SDO mutant on Ashdown agar at day 4 was examined using a morphotyping algorithm [26]. Bacterial structure was determined under light microscopy (Gram stain) and electron microscopy. The ability of the B. pseudomallei SDO mutant to invade A549 cells and survive in infected J774A.1 cells was measured as previously described [51], and compared with the wild type strain.

In the invasion efficiency assay, an A549 cell line was infected with culture of B. pseudomallei in LB broth containing 0,150 , or $300 \mathrm{mM} \mathrm{NaCl}$ at a multiplicity of infection (MOI) of 100 for $1 \mathrm{hr}$ to bring bacteria into contact with the cells and allow bacterial entry. The monolayers were overlaid with a medium containing $250 \mu \mathrm{g} / \mathrm{ml}$ kanamycin (Gibco) to kill extracellular bacteria for $1 \mathrm{hr}$. Viable intracellular bacteria were released from the infected cells at $4 \mathrm{hrs}$ post-infection by lysis with $0.5 \%$ Triton X-100 (Sigma-Aldrich), and then plated on Trypticase soy agar. Colony forming units were measured after $36-48 \mathrm{hrs}$ of incubation at $37^{\circ} \mathrm{C}$. The percentage of invasion efficiency is calculated as the number of intracellular bacteria at $4 \mathrm{hrs}$ postinfection $\times 100$ and divided by the CFU added.

For the intracellular survival assay, a J774A.1 cell line was inoculated with culture of $B$. pseudomallei in LB broth containing 0,150 , or $300 \mathrm{mM} \mathrm{NaCl}$ at a multiplicity of infection (MOI) of 2 for 2 hrs to allow bacterial entry. After infection for $2 \mathrm{hrs}$, a medium containing $250 \mu \mathrm{g} / \mathrm{ml}$ kanamycin was added to kill extracellular bacteria. The cell culture was incubated for $2 \mathrm{hrs}$ to completely eliminate residual extracellular bacteria. An additional incubation was then performed; infected cells were covered with a medium containing $20 \mu \mathrm{g} / \mathrm{ml}$ kanamycin to inhibit the growth of the remaining extracellular bacteria. After 4, 6, and 8 hrs post-infection, the cell monolayer was washed with pre-warmed PBS and lysed with $100 \mu \mathrm{l}$ of $0.1 \%$ Triton X-100 (Sigma Chemical Co.) in distilled water. Intracellular bacteria were quantitated by dilution and plated on Trypticase soy agar. The bacterial colonies were counted after $36 \mathrm{hrs}$ of incubation at $37^{\circ} \mathrm{C}$. The percentage of intracellular survival was determined by the following equation: (number of intracellular bacteria post-infection $\times 100$ )/ number of CFU added.

\section{Determination of the $B$. pseudomallei survival under oxidative stresses}

The survival of $B$. pseudomallei in oxidative conditions was determined by the growth on oxidant agar plates. The 6 hrs cultures of B. pseudomallei in LB broth containing 0,150 , or $300 \mathrm{mM} \mathrm{NaCl}$ were washed and resuspended with PBS. The bacterial concentration was adjusted to $\mathrm{OD}_{600}$ 0.15. A 10-fold dilution of the inoculums was performed. Ten microlitres of all dilutions of bacteria in PBS were spotted onto the LB agar with and without adding sub-lethal concentrations of menadione $(400 \mu \mathrm{M}), \mathrm{H}_{2} \mathrm{O}_{2}$ $(250 \mu \mathrm{M})$ and $\mathrm{tBOOH}(200 \mu \mathrm{M})$ [52]. Colony counts were performed after incubation at $37^{\circ} \mathrm{C}$ for $24 \mathrm{hrs}$. The number of colonies on plates containing oxidants was compared with that on control plates (LB agar without oxidant) and presented as \% bacterial survival. \% Survival = CFU (with oxidant) $\times 100 /$ CFU (without oxidant) .

\section{Statistical analysis}

All assays were conducted in triplicate, and unpaired $t$-test of independent experiments was performed by statistical analysis using GraphPad Prism 6 program (STATCON). Results were considered significant at $p$-value $\leq 0.05$.

\section{Additional file}

Additional file 1: Construction and verification of $B$. pseudomallei SDO mutant. A) A 566 bp DNA fragment containing 298 bp-upstream and 288 bp-downstream of the SDO gene was replaced into the B. pseudomallei K96243 genome using the pEXKm5-based allele replacement system [19]. B) PCR of B. pseudomallei wild type, SDO mutant and SDO complement strain 
were performed with the BPSS2242-F1 and BPSS2242-R2 primer pair (lane 1: 100-3000 bp marker ladder; lane 2: negative control; lane 3: K96243; lane 4: SDO mutant; and lane 5: SDO complement strain). C) PCR analysis of pEXKm5 plasmid backbone within the $B$. pseudomallei genome using oriT specific primers (lane 1: 100-3000 bp marker ladder; lane 2: negative control; lane 3: SDO mutant before sucrose selection; lane 4: SDO complement strain before sucrose selection; lane 5: SDO mutant after sucrose selection; and lane 6: SDO complement strain after sucrose selection).

\section{Competing interests}

The authors declare that they have no competing interests.

\section{Authors' contributions}

PP and NC designed the research. UB performed bioinformatics analysis of protein sequences. PP and ST constructed the mutant. PP and PP constructed the complementary strain. PP and BU carried out enzymatic activity assay. PP and VM carried out growth kinetic assay. PP and TS performed the colony examination. PP and PP carried out invasion and survival assay. PP and MV performed the oxidative response experiment. PP carried out statistical analysis. PP wrote the manuscript. NC and KS critically revised the manuscript for intellectual content. All authors read and approved the final version of the manuscript.

\section{Acknowledgements}

This work was supported by a Research Grant from the Faculty of Tropical Medicine, Mahidol University, Fiscal year 2011. NC is supported by a Wellcome Trust Career Development Award in Public Health and Tropical Medicine, UK (Grant: 087769/Z/08/Z). We thank Herbert P. Schweizer for providing pEXKm5 vector. We thank Prof. Srisin Khusmith for her insightful advice, and Mr. Glad Rotaru \& Mr. Paul Adams, of the Office of Research Services, Faculty of Tropical Medicine, Mahidol University, for proof-reading the manuscript.

\section{Author details}

'Department of Microbiology and Immunology, Faculty of Tropical Medicine, Mahidol University, Bangkok 10400, Thailand. ${ }^{2}$ Department of Molecular Tropical Medicine and Genetics, Faculty of Tropical Medicine, Mahidol University, Bangkok 10400, Thailand. 'epartment of Immunology, Faculty of Medicine, Siriraj Hospital, Mahidol University, Bangkok 10700, Thailand. ${ }^{4}$ Mahidol-Oxford Tropical Medicine Research Unit, Faculty of Tropical Medicine, Mahidol University, Bangkok 10400, Thailand.

Received: 10 September 2013 Accepted: 21 November 2013 Published: 2 January 2014

\section{References}

1. White NJ: Melioidosis. Lancet 2003, 361:1715-1722

2. Currie BJ, Jacups SP: Intensity of rainfall and severity of melioidosis, Australia. Emerg Infect Dis 2003, 9:1538-1542.

3. Leelarasamee A, Trakulsomboon S, Kusum M, Dejsirilert S: Isolation rates of Burkholderia pseudomallei among the four regions in Thailand. Southeast Asian J Trop Med Public Health 1997, 28:107-113.

4. Vuddhakul V, Tharavichitkul P, Na-Ngam N, Jitsurong S, Kunthawa B, Noimay P, Noimay P, Binla A, Thamlikitkul V: Epidemiology of Burkholderia pseudomallei in Thailand. Am J Trop Med Hyg 1999, 60:458-461.

5. Smith JJ, Travis SM, Greenberg EP, Welsh MJ: Cystic fibrosis airway epithelia fail to kill bacteria because of abnormal airway surface fluid. Cell 1996, 85:229-236

6. Joris L, Dab I, Quinton PM: Elemental composition of human airway surface fluid in healthy and diseased airways. Am Rev Respir Dis 1993, 148:1633-1637.

7. Vandamme $\mathrm{P}$, Holmes $\mathrm{B}$, Vancanneyt $\mathrm{M}$, Coenye $\mathrm{T}$, Hoste $\mathrm{B}$, Coopman $\mathrm{R}$, Revets H, Lauwers S, Gillis M, Kersters K, et al: Occurrence of multiple genomovars of Burkholderia cepacia in cystic fibrosis patients and proposal of Burkholderia multivorans sp. nov. Int J Syst Bacteriol 1997, 47:1188-1200.

8. Mahenthiralingam E, Baldwin A, Vandamme P: Burkholderia cepacia complex infection in patients with cystic fibrosis. J Med Microbiol 2002, 51:533-538.
9. O'Carroll MR, Kidd TJ, Coulter C, Smith HV, Rose BR, Harbour C, Bell SC: Burkholderia pseudomallei: another emerging pathogen in cystic fibrosis. Thorax 2003, 58:1087-1091.

10. O'Quinn AL, Wiegand EM, Jeddeloh JA: Burkholderia pseudomallei kills the nematode Caenorhabditis elegans using an endotoxin-mediated paralysis. Cell Microbiol 2001, 3:381-393.

11. Pumirat $P$, Cuccui J, Stabler RA, Stevens JM, Muangsombut V, Singsuksawat E, Stevens MP, Wren BW, Korbsrisate S: Global transcriptional profiling of Burkholderia pseudomallei under salt stress reveals differential effects on the Bsa type III secretion system. BMC Microbiol 2010, 10:171.

12. Pumirat $P$, Saetun $P$, Sinchaikul $S$, Chen ST, Korbsrisate $S$, Thongboonkerd V: Altered secretome of Burkholderia pseudomallei induced by salt stress. Biochim Biophys Acta 2009, 1794:898-904.

13. Bhatt $\mathrm{S}$, Weingart $\mathrm{CL}$ : Identification of sodium chloride-regulated genes in Burkholderia cenocepacia. Curr Microbiol 2008, 56:418-422.

14. Holden MT, Titball RW, Peacock SJ, Cerdeno-Tarraga AM, Atkins T, Crossman LC, Pitt T, Churcher C, Mungall K, Bentley SD, et al: Genomic plasticity of the causative agent of melioidosis, Burkholderia pseudomallei. Proc Natl Acad Sci U S A 2004, 101:14240-14245.

15. Altschul SF, Gish W, Miller W, Myers EW, Lipman DJ: Basic local alignment search tool. J Mol Biol 1990, 215:403-410.

16. Larkin MA, Blackshields G, Brown NP, Chenna R, McGettigan PA, McWilliam H, Valentin F, Wallace IM, Wilm A, Lopez R, et al: Clustal W and Clustal X version 2.0. Bioinformatics 2007, 23:2947-2948.

17. Schwede T, Kopp J, Guex N, Peitsch MC: SWISS-MODEL: An automated protein homology-modeling server. Nucleic Acids Res 2003, 31:3381-3385.

18. Laskowski RA, MacArthur MW, Moss DS, Thornton JM: PROCHECK: a program to check the stereochemical quality of protein structures. J App/ Cryst 1993, 26:283-291.

19. Lopez CM, Rholl DA, Trunck LA, Schweizer HP: Versatile dual-technology system for markerless allele replacement in Burkholderia pseudomallei. Appl Environ Microbiol 2009, 75:6496-6503.

20. Pauly HE, Pfleiderer G: D-glucose dehydrogenase from Bacillus megaterium M 1286: purification, properties and structure. Hoppe Seylers Z Physiol Chem 1975, 356:1613-1623.

21. Pruksachartvuthi S, Aswapokee N, Thankerngpol K: Survival of Pseudomonas pseudomallei in human phagocytes. J Med Microbiol 1990, 31:109-114.

22. Jones AL, Beveridge TJ, Woods DE: Intracellular survival of Burkholderia pseudomallei. Infect Immun 1996, 64:782-790.

23. Brown SA, Whiteley M: Characterization of the L-lactate dehydrogenase from Aggregatibacter actinomycetemcomitans. PLoS One 2009, 4:e7864.

24. Pruss BM, Nelms JM, Park C, Wolfe AJ: Mutations in NADH:ubiquinone oxidoreductase of Escherichia coli affect growth on mixed amino acids. J Bacteriol 1994, 176:2143-2150.

25. Rodriguez-Montelongo L, Volentini SI, Farias RN, Massa EM, Rapisarda VA The $\mathrm{Cu}$ (II)-reductase NADH dehydrogenase-2 of Escherichia coli improves the bacterial growth in extreme copper concentrations and increases the resistance to the damage caused by copper and hydroperoxide. Arch Biochem Biophys 2006, 451:1-7.

26. Chantratita N, Wuthiekanun V, Boonbumrung K, Tiyawisutsri R, Vesaratchavest M, Limmathurotsakul D, Chierakul W, Wongratanacheewin S, Pukritiyakamee S, White NJ, et al: Biological relevance of colony morphology and phenotypic switching by Burkholderia pseudomallei. J Bacteriol 2007, 189:807-817.

27. Fu HS, Hassett DJ, Cohen MS: Oxidant stress in Neisseria gonorrhoeae: adaptation and effects on L-(+)-lactate dehydrogenase activity. Infect Immun 1989, 57:2173-2178.

28. Liu L, Hausladen A, Zeng M, Que L, Heitman J, Stamler JS, Steverding D: Nitrosative stress: protection by glutathione-dependent formaldehyde dehydrogenase. Redox Rep 2001, 6:209-210.

29. Messner KR, Imlay JA: Mechanism of superoxide and hydrogen peroxide formation by fumarate reductase, succinate dehydrogenase, and aspartate oxidase. J Biol Chem 2002, 277:42563-42571.

30. Cabiscol E, Tamarit J, Ros J: Oxidative stress in bacteria and protein damage by reactive oxygen species. Int Microbiol 2000, 3:3-8.

31. Weerakoon DR, Borden NJ, Goodson CM, Grimes J, Olson JW: The role of respiratory donor enzymes in Campylobacter jejuni host colonization and physiology. Microb Pathog 2009, 47:8-15. 
32. Miller JL, Velmurugan $\mathrm{K}$, Cowan MJ, Briken V: The type I NADH dehydrogenase of Mycobacterium tuberculosis counters phagosomal NOX2 activity to inhibit TNF-alpha-mediated host cell apoptosis. PLOS Pathog 2010 6:e1000864.

33. Hoper D, Volker U, Hecker M: Comprehensive characterization of the contribution of individual SigB-dependent general stress genes to stress resistance of Bacillus subtilis. J Bacteriol 2005, 187:2810-2826.

34. Widdicombe $\mathrm{JH}$ : Altered $\mathrm{NaCl}$ concentration of airway surface liquid in cystic fibrosis. Pflugers Arch 2001, 443(Suppl 1):S8-S10.

35. Yamamoto $\mathrm{T}$ : Stress response of pathogenic bacteria-are stress proteins virulence factors? Nihon Saikingaku Zasshi 1996, 51:1025-1036.

36. Inglis TJ, Sagripanti JL: Environmental factors that affect the survival and persistence of Burkholderia pseudomallei. Appl Environ Microbiol 2006, 72:6865-6875

37. Robertson J, Levy A, Sagripanti JL, Inglis TJ: The survival of Burkholderia pseudomallei in liquid media. Am J Trop Med Hyg 2010, 82:88-94.

38. Jornvall H, Persson B, Krook M, Atrian S, Gonzalez-Duarte R, Jeffery J, Ghosh D: Short-chain dehydrogenases/reductases (SDR). Biochemistry 1995, 34:6003-6013.

39. Rodrigues F, Sarkar-Tyson M, Harding SV, Sim SH, Chua HH, Lin CH, Han X, Karuturi RK, Sung K, Yu K, et al: Global map of growth-regulated gene expression in Burkholderia pseudomallei, the causative agent of melioidosis. J Bacteriol 2006, 188:8178-8188.

40. Purves J, Cockayne A, Moody PC, Morrissey JA: Comparison of the regulation, metabolic functions, and roles in virulence of the glyceraldehyde-3-phosphate dehydrogenase homologues gapA and gapB in Staphylococcus aureus. Infect Immun 2010, 78:5223-5232.

41. Laouami S, Messaoudi K, Alberto F, Clavel T, Duport C: Lactate dehydrogenase $A$ promotes communication between carbohydrate catabolism and virulence in Bacillus cereus. J Bacteriol 2011, 193:1757-1766

42. Jagadeesan B, Koo OK, Kim KP, Burkholder KM, Mishra KK, Aroonnual A, Bhunia AK: LAP, an alcohol acetaldehyde dehydrogenase enzyme in Listeria, promotes bacterial adhesion to enterocyte-like Caco-2 cells only in pathogenic species. Microbiology 2010, 156:2782-2795.

43. Venugopal A, Bryk R, Shi S, Rhee K, Rath P, Schnappinger D, Ehrt S, Nathan C: Virulence of Mycobacterium tuberculosis depends on lipoamide dehydrogenase, a member of three multienzyme complexes. Cell Host Microbe 2011, 9:21-31.

44. Brzezinska M, Szulc I, Brzostek A, Klink M, Kielbik M, Sulowska Z, Pawelczyk J, Dziadek J: The role of 3-ketosteroid 1(2)-dehydrogenase in the pathogenicity of Mycobacterium tuberculosis. BMC Microbiol 2013, 13:43.

45. Bijtenhoorn P, Mayerhofer H, Müller-Dieckmann J, Utpatel C, Schipper C, Hornung C, Szesny M, Grond S, Thürmer A, Brzuszkiewicz E, et al: A novel metagenomic short-chain dehydrogenase/reductase attenuates Pseudomonas aeruginosa biofilm formation and virulence on Caenorhabditis elegans. PLoS One 2011, 6:e26278.

46. Burtnick MN, Brett PJ, Nair V, Warawa JM, Woods DE, Gherardini FC: Burkholderia pseudomallei type III secretion system mutants exhibit delayed vacuolar escape phenotypes in RAW 264.7 murine macrophages. Infect Immun 2008, 76:2991-3000.

47. Muangsombut $V$, Suparak S, Pumirat P, Damnin S, Vattanaviboon P, Thongboonkerd $V$, Korbsrisate S: Inactivation of Burkholderia pseudomallei bsaQ results in decreased invasion efficiency and delayed escape of bacteria from endocytic vesicles. Arch Microbiol 2008, 190:623-631.

48. Seib KL, Wu HJ, Kidd SP, Apicella MA, Jennings MP, McEwan AG: Defenses against oxidative stress in Neisseria gonorrhoeae: a system tailored for a challenging environment. Microbiol Mol Biol Rev 2006, 70:344-361.

49. Seib KL, Tseng HJ, McEwan AG, Apicella MA, Jennings MP: Defenses against oxidative stress in Neisseria gonorrhoeae and Neisseria meningitidis: distinctive systems for different lifestyles. J Infect Dis 2004, 190:136-147.

50. Chantratita N, Tandhavanant S, Wikraiphat C, Trunck LA, Rholl DA Thanwisai A, Saiprom N, Limmathurotsakul D, Korbsrisate S, Day NP, et al: Proteomic analysis of colony morphology variants of Burkholderia pseudomallei defines a role for the arginine deiminase system in bacterial survival. J Proteomics 2012, 75:1031-1042.

51. Suparak S, Kespichayawattana W, Haque A, Easton A, Damnin S, Lertmemongkolchai G, Bancroft GJ, Korbsrisate S: Multinucleated giant cell formation and apoptosis in infected host cells is mediated by
Burkholderia pseudomallei type III secretion protein BipB. J Bacteriol 2005, 187:6556-6560.

52. Vattanaviboon P, Panmanee W, Mongkolsuk S: Induction of peroxide and superoxide protective enzymes and physiological cross-protection against peroxide killing by a superoxide generator in Vibrio harveyi. FEMS Microbiol Lett 2003, 221:89-95.

doi:10.1186/1471-2180-14-1

Cite this article as: Pumirat et al:: The role of short-chain dehydrogenase/ oxidoreductase, induced by salt stress, on host interaction of B. pseudomallei. BMC Microbiology 2014 14:1.

\section{Submit your next manuscript to BioMed Central and take full advantage of:}

- Convenient online submission

- Thorough peer review

- No space constraints or color figure charges

- Immediate publication on acceptance

- Inclusion in PubMed, CAS, Scopus and Google Scholar

- Research which is freely available for redistribution

Submit your manuscript at www.biomedcentral.com/submit
C) Biomed Central 